\title{
Status of the EDELWEISS Experiment
}

\author{
Presented by Philippe Di Stefano, CEA/Saclay, DSM/DAPNIA/SPP

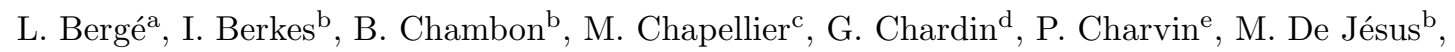

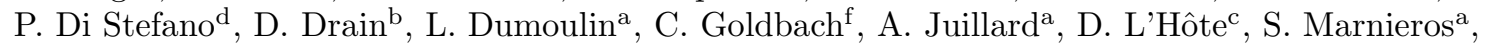

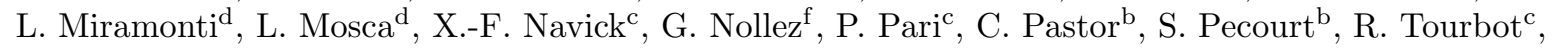 \\ D. Yvon ${ }^{\mathrm{d}}$
}

${ }^{a}$ Centre de Spectrométrie Nucléaire et Spectrométrie de Masse, IN2P3-CNRS, Univ. Paris XI, Bat. 108, F-91405 Orsay Cedex, France

${ }^{\mathrm{b}}$ Institut de Physique Nucléaire de Lyon and Université Claude Bernard, Lyon I, IN2P3-CNRS, 43 Bd. du 11 novembre 1918, F-69622 Villeurbanne Cedex, France

${ }^{\mathrm{c}}$ CEA, Centre d'Etudes de Saclay, DSM/DRECAM, Service de Physique de l'Etat Condensé, F-91191 Gif-sur-Yvette Cedex, France

${ }^{\mathrm{d} C E A}$, Centre d'Etudes de Saclay, DSM/DAPNIA, Service de Physique des Particules, F-91191 Gif-sur-Yvette Cedex, France

eLaboratoire Souterrain de Modane, CEA-CNRS, 90 Rue Polset, F-73500 Modane, France

${ }^{\mathrm{f}}$ Institut d'Astrophysique de Paris, INSU-CNRS, 98bis Bd. Arago, F-75014 Paris, France

\begin{abstract}
The status of the EDELWEISS experiment (underground dark matter search with heat-ionisation bolometers) is reviewed. Auspicious results achieved with a prototype $70 \mathrm{~g}$ Ge heat-ionisation detector under a $2 \mathrm{~V}$ reverse bias tension are discussed. Based on gamma and neutron calibrations, a best-case rejection factor, over the 15-45 keV range, of $99.7 \%$ for gammas, with an acceptance of $94 \%$ for neutrons, is presented first. Some operational results of physical interest obtained under poor low radioactivity conditions follow. They include a raw event rate of around 30 events $/$ day $/ \mathrm{kg} / \mathrm{keV}$ over the same energy range, and, after rejection of part of the background, lead to a conservative upper limit on the signal of approximately 1.6 events $/$ day $/ \mathrm{kg} / \mathrm{keV}$ at a $90 \%$ confidence level. Performance degrading surface effects of the detector are speculated upon; and planned upgrades are summarized.
\end{abstract}

\section{DARK MATTER AND DIRECT DE- TECTION}

Ironically enough, the problem of dark matter has been glaring in physics for several years now [1.2], and the case for a non-baryonic contribution to it is compelling [3]. Moreover, structure formation suggests a sizable amount of cold dark matter made up of particles in the $\mathrm{GeV}$ to $\mathrm{TeV}$ range. Various supersymmetric theories generate a plausible candidate for WIMPs, the Lightest Supersymmetric Particle, which would be stable in the case of R-parity conservation [4. Several searches for these WIMPs are un- derway, using either indirect means (i.e. looking for products of WIMP annihilation) or direct ones [5]. The latter, first suggested by Goodman and Witten [6], seek interactions of WIMPs themselves in detectors, which historically have been semi-conducting devices, scintillators and, more recently, bolometers. Bolometers are attractive for several reasons : they achieve excellent energy resolutions and low thresholds, and can be fashioned from various materials. Furthermore, if made of $\mathrm{Ge}$ or $\mathrm{Si}$, they should be easily cleansed of radioactive impurities, and can reject a large fraction of background noise thanks to the possibility of simultaneous heat and ionisation mea- 
surement [7]. The EDELWEISS experiment, presented hereafter, has developed and tested such bolometers.

\section{THE EDELWEISS EXPERIMENT AT THE LABORATOIRE SOUTERRAIN DE MODANE}

EDELWEISS (Expérience pour DEtecter Les WIMPs En SIte Souterrain) is an underground direct WIMP detection experiment. The underground site is the Laboratoire Souterrain de Modane (LSM), located by a highway tunnel beneath the Franco-Italian Alps. The mountain provides protection equivalent to 4800 meters of water, which yields a six order of magnitude reduction in the cosmic muon flux. Natural radioactivity of the surrounding rock generates $4.10^{-6} / \mathrm{s} / \mathrm{cm}^{2}$ worth of fast neutrons. The importance of this value lies in the fact that neutrons, which interact a priori like WIMPs, are expected to make up the ultimate background noise for bolometers.

The initial experimental setup has been described in Ref. [8]. Since then, several enhancements have been made. Specifically, the dilution refrigerator now reaches temperatures as low as $10 \mathrm{mK}$, with a cooling power of $100 \mu \mathrm{W}$ at $100 \mathrm{mK}$; the electronics have undergone a straightforward upgrade since Ref. [9]; and a new detector has been tested. For the purpose of these tests and data taking, the part of the low radioactivity shielding closest to the detector has been omitted, and no radon removal has been performed. Moreover, off-line data analysis is now performed using optimal filter techniques in the frequency domain [10].

\section{DETECTOR AND CALIBRATION}

Detector design is detailed in Ref. [11]. Performances are summarized in Table 11. They have been obtained under a bias tension of $-2 \mathrm{~V}$, and with a ${ }^{57} \mathrm{Co}$ source to perform energy calibration (Fig. 1). Though they are satisfactory, in the heat-ionisation plane, the $122 \mathrm{keV}$ peak is somewhat smeared. This is due to the incomplete charge collection of a minority of events in

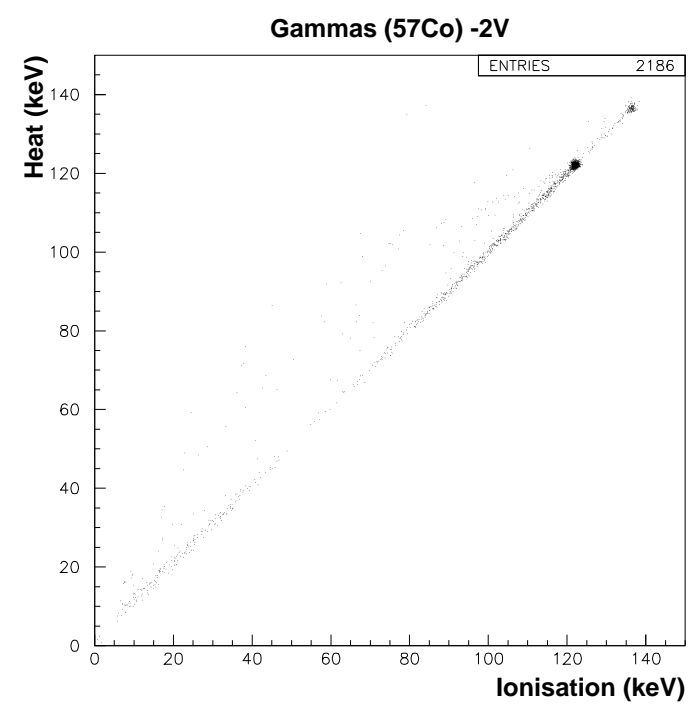

Figure 1. Energy calibration of detector using a ${ }^{57}$ Co source. Energies are normalized to equivalent electron recoils. Some off-axis events, including drooling from the $122 \mathrm{keV}$ peak, are visible. Smaller $136 \mathrm{keV}$ sibling appears also.

all likelihood near the surface of the detector, and will be discussed later on in this paper.

The distribution of gamma-induced recoil events has been studied further by means of a ${ }^{60} \mathrm{Co}$ source. This isotope has the advantage over

${ }^{57} \mathrm{Co}$ of emitting higher energy photons, less susceptible to surface effects in the detector, and providing a Compton-scattering plateau. With such a source, around $10 \%$ of events suffer from incomplete charge collection. Ignoring for the time

Table 1

Detector performance under a $-2 \mathrm{~V}$ bias tension. Ionisation energies are equivalent electron $\mathrm{keV}$.

\begin{tabular}{lcc}
\hline & Heat & Ionisation \\
\hline FWHM resolution at 122 keV & 1.2 & 1.25 \\
FWHM baseline noise & 0.85 & 1.1 \\
Effective energy threshold & 4 & 4 \\
\hline
\end{tabular}


being these pathological cases, the spread of the photon events, obtained by studying the ionisation to heat ratio in the 5 to $200 \mathrm{keV}$ heat range, is compatible with the assumptions that, over the studied energy scale, the resolutions of both channels are constant, the channel fluctuations are uncorrelated, and that at a given energy the dispersion of each channel is a gaussian variable. A slight systematic overestimation of the ionisation at low energies is under investigation.

The distribution of neutron-induced recoil events in heat-ionisation space is obtained using a ${ }^{252} \mathrm{Cf}$ source, as done previously [12,13]. Less statistics are available than with the photon source; moreover, because of the forward scattering of the neutrons on heavy nuclei which lowers the recoil energies, this study only covers the 5 to $50 \mathrm{keV}$ heat interval. The spread of the ionisation over heat distribution shows a widening as heat increases. This is being compared to theoretical predictions [14]. The neutron zone that will be of use in Section 1 is defined in ionisation over heat space, and for each heat bin, as being within three standard deviations of the mean of the distribution.

Using the ${ }^{252} \mathrm{Cf}$ and ${ }^{60} \mathrm{Co}$ calibrations, it is possible to obtain a best case rejection factor for gammas and acceptance of neutron-type events in the detector. Over the 15 to $45 \mathrm{keV}$ heat spread, the rejection of gammas is $99.7 \pm 0.2 \%$ for an acceptance of neutrons amounting to $94 \pm 6 \%$, which yields a separation quality factor $Q$ (defined as per Ref. 15]) of $0.004 \pm 0.002$, as shown in Fig. 2 . Though impressive, it must be stressed that this is a best-case result only, since, in practice, current real data runs contain proportionately more low energy events which are more prone to suffer from surface effects in the detector. The ensuing incomplete charge collection pollutes the population divide as will be observed below.

\section{PHYSICS RESULTS}

Nearly twenty different physics runs taken over late July and early August have been compiled, amounting to $0.65 \mathrm{~kg}$.days. For these runs, a $2 \mathrm{~V}$ reverse bias tension was applied to the detector, and only minimal radioactive shielding was
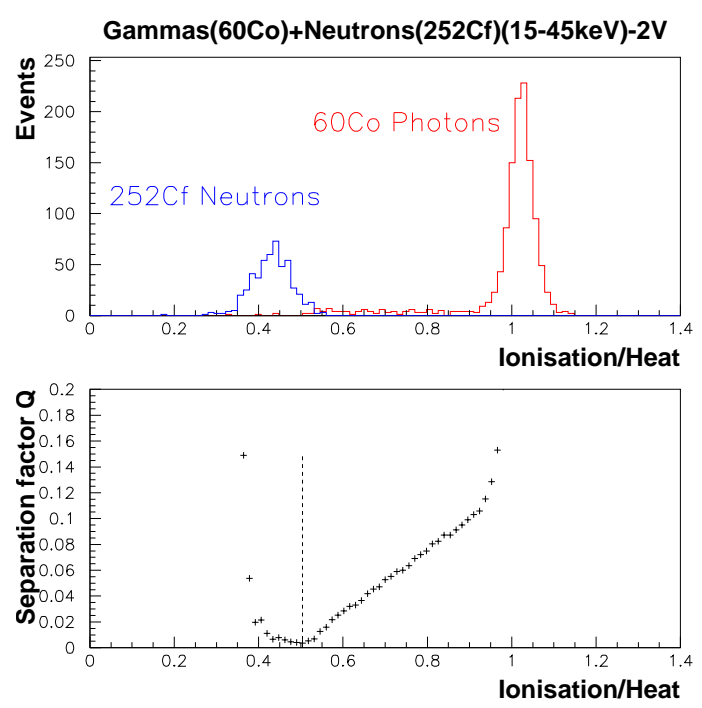

Figure 2. Best-case separation of electron recoils from nuclear recoils in the $15-45 \mathrm{keV}$ heat range, under $-2 \mathrm{~V}$ of bias tension. Top graph shows distribution of the ionisation to heat ratio for two calibration populations of photons and neutrons, from a ${ }^{60} \mathrm{Co}$ and a ${ }^{252} \mathrm{Cf}$ source respectively. Bottom graph presents evolution of separation quality factor $Q$ as a function of the aforementioned ratio; vertical dashed line marks minimum.

in place: neither Roman lead close shield, nor any form of radon removal. An unsurprisingly high raw rate of around 30 events $/$ day $/ \mathrm{kg} / \mathrm{keV}$, is therefore obtained in the 15 to $45 \mathrm{keV}$ range as shown in Fig. 3. Moreover, summary inspection of the data shows a gamma peak at approximately $47 \mathrm{keV}$. This can be attributed to photons from ${ }^{210} \mathrm{~Pb}$ pollution. Furthermore, a cursory glance at the distribution (top of Fig. (1) reveals that separation of nuclear and electron recoils has regressed with respect to the calibrations. Indeed, the distribution of the ionisation over heat ratio now clearly shows a third population, straddling the photons and the expected position of the neutrons. This reflects the incomplete charge collection of many low energy electron recoils which 

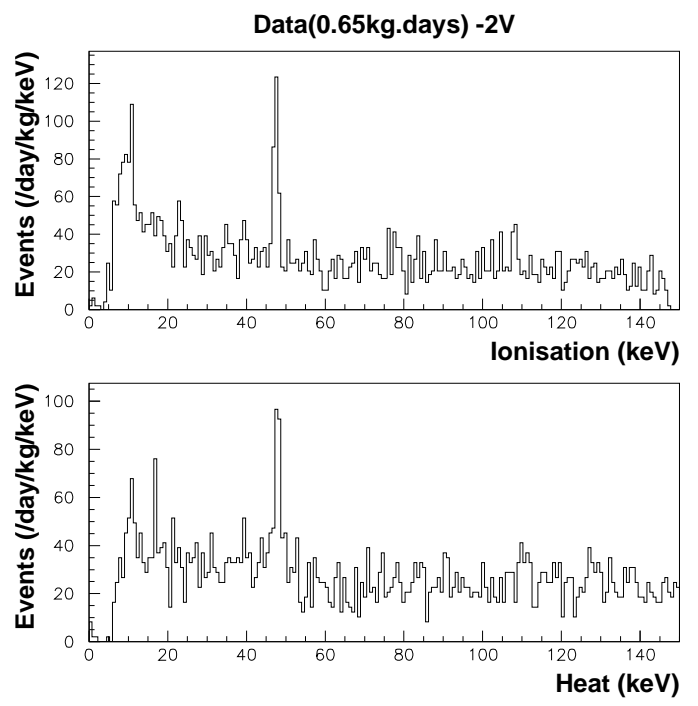

Figure 3. $0.65 \mathrm{~kg}$.days of raw data taken at a $2 \mathrm{~V}$ reverse bias tension. The $47 \mathrm{keV}$ peak of ${ }^{210} \mathrm{~Pb}$ stands out. Rate over $15-45 \mathrm{keV}$ range is approximately 30 events/day $/ \mathrm{kg} / \mathrm{keV}$.

therefore migrate towards the neutron zone.

To circumvent this, a cut is made, keeping only the lower half of the neutron zone in ionisation over heat space as defined in the previous section. The resulting spectrum, adjusted for the neutron acceptance of 0.5 now, leads to a rate of about 1 event $/$ day $/ \mathrm{kg} / \mathrm{keV}$ and an upper limit at a $90 \%$ confidence level of 1.6 events $/$ day $/ \mathrm{kg} / \mathrm{keV}$. This encouraging result remains undoubtedly overly cautious, as it assumes that none of the events in the neutron zone are due to poor charge collection of electron recoils.

Indeed if the shape of the background was known, it could be subtracted from the data and an optimal cut could be determined [15]. However, as Fig. 4 confirms, the ${ }^{60} \mathrm{Co}$ data clearly does not reflect the true background. Thus the data is itself used under the unverifiable assumption that it contains no signal. This affords less discrimination than possible in the best case, with an acceptance of $50 \pm 4 \%$, a rejection factor of
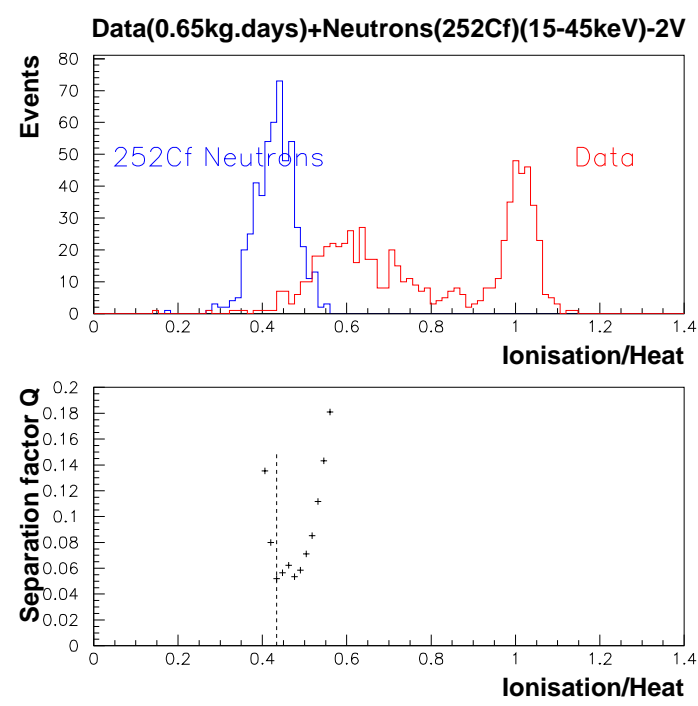

Figure 4. Real-life distribution of electron and nuclear recoils in ionisation over heat space, for the $15-45 \mathrm{keV}$ energy range. Top shows that background data contains a population of events falling between calibration neutrons and well behaved electron recoils. Bottom demonstrates further that populations have melded compared to the best-case scenario presented in Fig. 2 .

$98.7 \pm 0.4 \%$ and a separation factor $Q=0.05 \pm$ 0.02. These entail, still under the assumption that the experimental data contains no signal, an upper limit following 15] at the $90 \%$ conf. level of 0.5 events $/$ day $/ \mathrm{kg} / \mathrm{keV}$ for the $15-45 \mathrm{keV}$ range. This includes a contribution from the statistical uncertainty of the neutron calibration amounting to 0.04 events $/$ day $/ \mathrm{kg} / \mathrm{keV}$. The discrepancy between the result cited in the previous paragraph and that just mentioned is linked to the assumption made in the latter. In the future, strongly increased statistics should enable a more adequate method of analysis based on a weaker hypothesis. 


\section{SURFACE EFFECTS AND INFLU- ENCE OF BIAS TENSION}

Despite the promising results just demonstrated, off-axis events evidently hinder the rejection capabilities of this type of detector. Incomplete charge collection would account for events displaying only ionisation deficiency. Incomplete charge collection coupled to the Luke-Neganov effect (enhancement of heat signal by the process of collecting charges [16]) can explain the nonhorizontal drooling observed for instance around the $122 \mathrm{keV}$ photoelectric peak of ${ }^{57} \mathrm{Co}$ (Fig. 1). These effects are expected to affect mainly events occurring near the surface of the detector, and can be described as loss of part of the backdiffused charge when it nears the surface [17,18]. The third population, lying between neutrons and gammas in the ionisation over heat distribution, could thus be understood as being superficial events, in all likelihood betas, or low energy $\mathrm{X}$-rays or gammas. Identification and elimination of the sources of these low energy events is a priority. That said events manage to make it to the detector implies they may originate from its immediate vicinity. As a matter of fact, the NTD thermometer is itself a prime suspect. In addition, work is underway to further study effects on the off-axis events of bias tensions down to $-12 \mathrm{~V}$, as there is some hope of improvement since a stronger electric field might be more efficient in collecting charges deposited near the surface 18]. Such high bias tensions are acceptable in the current detector because of its p-i-n design.

\section{PERSPECTIVES FOR EDELWEISS}

Analysis work will continue on the $-2 \mathrm{~V}$ bias tension data as well as on $1.25 \mathrm{~kg}$.days of more recent $-6 \mathrm{~V}$ data. Regarding the current experimental setup, much effort is now being put into reducing its radioactive background. This includes investigating all surfaces close to the detector as well as the bolometer itself, and in particular the NTD thermometer. Eliminating to a great extent any background from the latter will be possible shortly by switching to resistive thermometers made of NbSi thin-films [19]. Moreover, two additional bolometers of a design similar to that of the current one should be installed in the near term at the LSM, followed by three $350 \mathrm{~g}$ scaled up devices. Lastly, planning is underway for the cryogenic infrastructure and acquisition logistics of a $10 \mathrm{~kg}$ array of detectors.

\section{ACKNOWLEDGEMENTS}

The collaboration as a whole wish to thank the staff of the LSM for their efficient support. The speaker is also grateful to J. Mallet and J. Rich (DAPNIA/SPP) for timely and useful discussion.

\section{REFERENCES}

1. D. Schramm, these Proceedings

2. L. Mosca, Surveys in High Energy Phys. 9 (1996) 275

3. L. Bergstrom, these Proceedings

4. G. Jungman et al., Phys. Rep. 267 (1996) 195

5. D. Caldwell, these Proceedings

6. M.W. Goodman and E. Witten, Phys. Rev. D 31 (1985) 3059

7. T. Shutt et al., Phys. Rev. Let. 69 (1992) 3425

8. A. de Bellefon et al., Astroparticle Phys. 6 (1996) 35

9. D. Yvon, Nucl. Instr. Meth. Phys. Res. A 368 (1996) 778

10. S.H. Moseley et al., J. Appl. Phys. 56 (1984) 1257

11. D. L'Hôte et al., Proc. 7th Int. Work. on Low Temp. Det. (1997), ISBN 3-00-002266-X, 240

12. T. Shutt et al., Nucl. Instr. Meth. Phys. Res. A 370 (1996) 165

13. D. L'Hôte et al., Proc. 7th Int. Work. on Low Temp. Det. (1997), ISBN 3-00-002266-X, 237

14. J. Lindhard et al., Mat. Fys. Medd. Dan. Vid. Selsk. 33, Num. 14 (1963)

15. R.J. Gaitskell et al., Nucl. Phys. B (Proc. Suppl.) 51B (1996) 279

16. P.N. Luke et al., Nucl. Instr. Meth. Phys. Res. A 289 (1990) 406

17. M.J. Penn et al., Nucl. Instr. Meth. Phys. Res. A 370 (1996) 215

18. T. Shutt et al., Proc. 7th Int. Work. on Low Temp. Det. (1997), ISBN 3-00-002266-X, 224

19. S. Marnieros et al., ibid., 134 